TRANSACTIONS OF THE

AMERICAN MATHEMATICAL SOCIETY

Volume 364, Number 7, July 2012, Pages 3513-3528

S 0002-9947(2012)05439-0

Article electronically published on February 27, 2012

\title{
AN ESTIMATE FOR THE SECTIONAL CURVATURE OF CYLINDRICALLY BOUNDED SUBMANIFOLDS
}

\author{
LUIS J. ALÍAS, G. PACELLI BESSA, AND J. FABIO MONTENEGRO
}

\begin{abstract}
We give sharp sectional curvature estimates for complete immersed cylindrically bounded $m$-submanifolds $\varphi: M^{m} \rightarrow N^{n-\ell} \times \mathbb{R}^{\ell}, n+\ell \leq 2 m-1$, provided that either $\varphi$ is proper with the norm of the second fundamental form with certain controlled growth or $M$ has scalar curvature with strong quadratic decay. The latter gives a non-trivial extension of the Jorge-Koutrofiotis Theorem. In the particular case of hypersurfaces, that is, $m=n-1$, the growth rate of the norm of the second fundamental form is improved. Our results will be an application of a generalized Omori-Yau Maximum Principle for the Hessian of a Riemannian manifold, in its newest elaboration given by Pigola, Rigoli and Setti (2005).
\end{abstract}

\section{INTRODUCTION}

Given complete Riemannian manifolds $M^{m}$ and $N^{n}$ with dimension $m<n$, the isometric immersion problem asks whether there exists an isometric immersion $\varphi: M \hookrightarrow N$. When $N^{n}=\mathbb{R}^{n}$ is the Euclidean space, the isometric problem is answered by the Nash Embedding Theorem that says that there is an isometric embedding $\varphi: M^{m} \hookrightarrow \mathbb{R}^{n}$, provided the codimension $n-m$ is sufficiently large; see 17. For small codimension, meaning in this paper that $n-m \leq m-1$, the answer in general depends on the geometries of $M$ and $N$. For instance, the HilbertEfimov Theorem [9], 12] says that no complete surface $M$ with sectional curvature $K_{M} \leq-\delta^{2}<0$ can be isometrically immersed in $\mathbb{R}^{3}$, and a classical result by C. Tompkins 24] states that a compact, flat, $m$-dimensional Riemannian manifold cannot be isometrically immersed in $\mathbb{R}^{2 m-1}$. Tompkins' result was extended in a series of papers by Chern and Kuiper [7, Moore [16], O'Neill [19], Otsuki [20] and Stiel [23, whose results can be summarized in the following theorem (we recall that a Cartan-Hadamard manifold is a simply connected, complete, Riemannian manifold with non-positive sectional curvatures).

Received by the editors June 11, 2010.

2010 Mathematics Subject Classification. Primary 53C42, 53C40.

Key words and phrases. Omori-Yau Maximum Principle, cylindrically bounded submanifolds, properly immersed submanifolds.

The first author's research was a result of the activity developed within the framework of the Programme in Support of Excellence Groups of the Región de Murcia, Spain, by Fundación Séneca, Regional Agency for Science and Technology (Regional Plan for Science and Technology 2007-2010). His research was partially supported by MEC project PCI2006-A7-0532, MICINN project MTM2009-10418, and Fundación Séneca project 04540/GERM/06, Spain.

The second author's research was partially supported by CNPq-CAPES (Brazil) and MEC project PCI2006-A7-0532 (Spain).

The third author's research was partially supported by CNPq-CAPES (Brazil). 
Theorem 1. Let $\varphi: M^{m} \hookrightarrow N^{n}, n \leq 2 m-1$, be an isometric immersion of a compact Riemannian $m$-manifold $M$ into a Cartan-Hadamard n-manifold $N$. Then the sectional curvatures of $M$ and $N$ satisfy

$$
\sup _{M} K_{M}>\inf _{N} K_{N}
$$

Theorem 1 was extended by Jorge and Koutrofiotis in [13] to bounded, complete submanifolds with scalar curvature bounded below and in the version presented by Pigola, Rigoli and Setti in [21, Theorem 1.15], with scalar curvature satisfying

$$
s_{M}(x) \geq-B^{2} \varrho_{M}^{2}(x) \cdot \prod_{j=1}^{k}\left(\log ^{(j)}\left(\varrho_{M}(x)\right)\right)^{2}, \varrho_{M}(x) \gg 1,
$$

for some constant $B>0$ and some integer $k \geq 1$, where $\varrho_{M}$ is the distance function on $M$ to a fixed point and $\log ^{(j)}$ is the $j$-th iterate of the logarithm.

Theorem 2 (Jorge-Koutrofiotis, [13]). Let $M^{m}$ and $N^{n}$ be complete Riemannian manifolds of dimensions $m$ and $n$, respectively, with $n \leq 2 m-1$ and let $\varphi: M \rightarrow N$ be an isometric immersion with $\varphi(M) \subset B_{N}(r)$, where $B_{N}(r)$ denotes a geodesic ball of $N$ centered at a point $p \in N$ and radius $r$. Assume that the radial sectional curvature $K_{N}^{\mathrm{rad}}$ along the radial geodesics issuing from $p$ satisfies $K_{N}^{\mathrm{rad}} \leq b$ in $B_{N}(r)$ and $0<r<\min \left\{\operatorname{inj}_{N}(p), \pi / 2 \sqrt{b}\right\}$, where we replace $\pi / 2 \sqrt{b}$ by $+\infty$ if $b \leq 0$. If the scalar curvature of $M$ satisfies (1.2), then

$$
\sup _{M} K_{M} \geq C_{b}^{2}(r)+\inf _{B_{N}(r)} K_{N},
$$

where

$$
C_{b}(t)= \begin{cases}\sqrt{b} \cot (\sqrt{b} t) & \text { if } b>0 \text { and } 0<t<\pi / 2 \sqrt{b}, \\ 1 / t & \text { if } b=0 \text { and } t>0 \\ \sqrt{-b} \operatorname{coth}(\sqrt{-b} t) & \text { if } b<0 \text { and } t>0 .\end{cases}
$$

Remark 3. If $N^{n}=\mathbb{N}^{n}(b)$ is the simply connected space form of constant sectional curvature $b$ and $M=\partial B_{\mathbb{N}^{n}(b)}(r) \subset \mathbb{N}^{n}(b)$ is a geodesic sphere of radius $r$, then equality in (1.3) is achieved.

The purpose of this paper is to extend the Jorge-Koutrofiotis Theorem to the case of complete cylindrically bounded submanifolds of a Riemannian product $N^{n-\ell} \times$ $\mathbb{R}^{\ell}$, where $N$ is a complete Riemannian manifold of dimension $n-\ell$. In this context, an isometric immersion $\varphi: M^{m} \rightarrow N^{n-\ell} \times \mathbb{R}^{\ell}$ of a Riemannian manifold $M^{m}$ is said to be cylindrically bounded if there exists $B_{N}(r)$, a geodesic ball of $N$ centered at a point $p \in N$ with radius $r>0$, such that $\varphi(M) \subset B_{N}(r) \times \mathbb{R}^{\ell}$ (see Figure 1).

In a recent paper [1, the two first authors, jointly with Dajczer, derived an estimate for the mean curvature $H$ of complete cylindrically bounded submanifolds into a product Riemannian manifold $N^{n-\ell} \times \mathbb{R}^{\ell}$. Specifically, it was proved in [1] that if the immersion $\varphi: M^{m} \rightarrow N^{n-\ell} \times \mathbb{R}^{\ell}$ is proper and $\varphi(M) \subset B_{N}(r) \times \mathbb{R}^{\ell}$, then

$$
\sup _{M}|H| \geq \frac{m-\ell}{m} C_{b}(r) .
$$

As a consequence, it follows from here that a complete hypersurface of given constant mean curvature lying inside a closed circular cylinder in Euclidean space cannot be proper if the circular bases is of sufficiently small radius (see Corollary 1 in [1). In particular, there exists no complete minimal hypersurface properly 


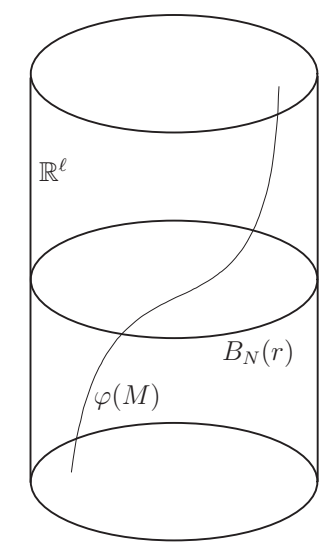

Figure 1. A cylindrically bounded submanifold.

immersed in $\mathbb{R}^{n}$ and having 2 (or more) bounded coordinates, showing that any possible counterexample to a conjecture of Calabi on complete minimal hypersurfaces [3] (see also [6]) cannot be proper.

Our main result here deals with the sectional curvature of such submanifolds, and it can be stated as follows.

Theorem 4. Let $M^{m}$ and $N^{n-\ell}$ be complete Riemannian manifolds of dimension $m$ and $n-\ell$ respectively, with $n+\ell \leq 2 m-1$. Let $\varphi: M^{m} \rightarrow N^{n-\ell} \times \mathbb{R}^{\ell}$ be a (cylindrically bounded) isometric immersion with $\varphi(M) \subset B_{N}(r) \times \mathbb{R}^{\ell}$. Assume that the radial sectional curvature $K_{N}^{\mathrm{rad}}$ along the radial geodesics issuing from $p$ satisfies $K_{N}^{\mathrm{rad}} \leq b$ in $B_{N}(r)$ and $0<r<\min \left\{\operatorname{inj}_{N}(p), \pi / 2 \sqrt{b}\right\}$, where we replace $\pi / 2 \sqrt{b}$ by $+\infty$ if $b \leq 0$. If either

(i) the scalar curvature of $M$ satisfies (1.2) or

(ii) $\varphi$ is proper and

$$
\sup _{\varphi^{-1}\left(B_{N}(r) \times \partial B_{\mathbb{R}}(t)\right)}\|\alpha\| \leq \sigma(t),
$$

where $\alpha$ is the second fundamental form of the immersion and $\sigma:[0,+\infty) \rightarrow \mathbb{R}$ is a positive function satisfying $\int_{0}^{+\infty} 1 / \sigma=+\infty$, then

$$
\sup _{M} K_{M} \geq C_{b}^{2}(r)+\inf _{B_{N}(r)} K_{N} .
$$

It is worth pointing out that the codimension restriction $n+\ell \leq 2 m-1$ cannot be relaxed. Actually, together with the bound $m \leq n-1$, it implies that $n-\ell \geq 3$ and $m \geq \ell+2$. In particular, for $n=3$ we have that $\ell=0$, and therefore $\varphi(M) \subset B_{N}(r)$. In fact, the flat cylinder $\mathbb{S}^{1}\left(r^{\prime}\right) \times \mathbb{R} \subset B_{\mathbb{R}^{2}}(r) \times \mathbb{R}$, for every $0<r^{\prime}<r$, shows that the restriction $2 m-1 \geq n+\ell$ is necessary.

On the other hand, our estimate (1.5) is sharp. Indeed, for every $n \geq 3$ and $\ell \leq n-3$ we can consider $M=\partial B_{\mathbb{N}^{n-\ell}(b)}\left(r^{\prime}\right) \times \mathbb{R}^{\ell}$ and take $\varphi: M \hookrightarrow B_{\mathbb{N}^{n-\ell}(b)}(r) \times \mathbb{R}^{\ell}$ to be the canonical isometric immersion for every $0<r^{\prime}<r$. Therefore $\sup _{M} K_{M}$ is the constant sectional curvature of the geodesic sphere $\partial B_{\mathbb{N}^{n-\ell}(b)}\left(r^{\prime}\right) \subset \mathbb{N}^{n-\ell}(b)$, 
which is given by

$$
\sup _{M} K_{M}=K_{\partial B_{\mathbb{N} n-\ell(b)}\left(r^{\prime}\right)}= \begin{cases}b / \sin ^{2}\left(\sqrt{b} r^{\prime}\right) & \text { if } b>0 \text { and } 0<r^{\prime}<\pi / 2 \sqrt{b}, \\ 1 / r^{2} & \text { if } b=0 \text { and } r^{\prime}>0, \\ -b / \sinh ^{2}\left(\sqrt{-b} r^{\prime}\right) & \text { if } b<0 \text { and } r^{\prime}>0 .\end{cases}
$$

In particular, observe that

$$
\sup _{M} K_{M}=K_{\partial B_{\mathbb{N}^{n-\ell}(b)}}\left(r^{\prime}\right)=C_{b}^{2}\left(r^{\prime}\right)+b .
$$

Since in this case $K_{\mathbb{N}^{n-\ell}(b)}=b$, then for every $0<r^{\prime}<r$ we have

$$
\sup _{M} K_{M}=C_{b}^{2}\left(r^{\prime}\right)+b \geq C_{b}^{2}(r)+\inf _{B_{\mathbb{N}^{n-\ell}(b)}(r)} K_{\mathbb{N}^{n-\ell}(b)},
$$

which shows that our estimate (1.5) is sharp.

Let $\varphi: M^{m} \rightarrow N^{n-\ell} \times \mathbb{R}^{\ell}$ be an isometric immersion of a compact Riemannian $m$-manifold $M^{m}$, and let $\pi_{N}: N^{n-\ell} \times \mathbb{R}^{\ell} \rightarrow N^{n-\ell}$ be the projection onto the first factor. Denote by $R_{M}$ the radius of the smallest ball of $N$ containing $\pi_{N}(\varphi(M))$. We will refer to $R_{M}$ as the extrinsic radius of the immersion. As a consequence of Theorem 4, we have the following versions of the Extrinsic Radius Theorem of Jorge-Xavier [14] (see also [2, Theorem 1.3]).

Corollary 5. Let $\varphi: M^{m} \rightarrow N^{n-\ell} \times \mathbb{R}^{\ell}$ be an isometric immersion of a compact Riemannian m-manifold $M^{m}$ into the product $N^{n-\ell} \times \mathbb{R}^{\ell}$ with $n+\ell \leq 2 m-1$, where $N^{n-\ell}$ is a complete Riemannian manifold with a pole and radial sectional curvature $K_{N}^{\mathrm{rad}} \leq b \leq 0$. Then, the extrinsic radius satisfies

$$
R_{M} \geq C_{b}^{-1}\left(\sqrt{\sup K_{M}-\inf K_{N}}\right) .
$$

In particular, if $N=\mathbb{R}^{n-\ell}$ we have that

$$
R_{M} \geq \frac{1}{\sqrt{\sup K_{M}}} .
$$

Corollary 6. Let $\varphi: M^{m} \rightarrow \mathbb{S}^{n-\ell} \times \mathbb{R}^{\ell}$ be an isometric immersion of a compact Riemannian $m$-manifold $M^{m}$ with $n+\ell \leq 2 m-1$. If $\sup K_{M} \leq 1$, then

$$
R_{M} \geq \pi / 2 \text {. }
$$

On the other hand, it is important to remark that for hypersurfaces, the growth rate of the norm of the second fundamental form in (1.4) can be improved as follows.

Theorem 7. Let $\varphi: M^{n-1} \rightarrow N^{n-\ell} \times \mathbb{R}^{\ell}$ be a properly immersed hypersurface with $\varphi(M) \subset B_{N}(r) \times \mathbb{R}^{\ell}, n-\ell \geq 3$. Suppose that $N$ satisfies the assumptions on the radial sectional curvatures as in Theorem 4 and the second fundamental form $\alpha$ satisfies

$$
\sup _{\varphi^{-1}\left(B_{N}(r) \times \partial B_{\mathbb{R} \ell}(t)\right)}\|\alpha\| \leq \sigma^{2}(t),
$$

where $\sigma:[0, \infty) \rightarrow \mathbb{R}$ is a positive function satisfying

$$
\int_{0}^{+\infty} \frac{1}{\sigma}=+\infty \quad \text { and } \quad \limsup _{t \rightarrow+\infty} \frac{1}{\sigma(t)}<+\infty .
$$

Then

$$
\sup _{M} K_{M} \geq C_{b}^{2}(r)+\inf _{B_{N}(r)} K_{N} .
$$


Remark 8. It should be remarked that Hasanis and Koutroufiotis [11] established similar sectional curvature estimates for cylindrically bounded submanifolds, with scalar curvature bounded below, of the Euclidean space $\mathbb{R}^{n}$. In a slightly more general situation, F. Giménez [10] established sectional curvature estimates for submanifolds with scalar curvature bounded below immersed in a tubular neighborhood of certain, ( $P$-submanifolds), embedded submanifolds of Hadamard manifolds. Our main results, besides extending Hasanis-Koutrofiotis results to a larger class of submanifolds, can be easily adapted to reproduce Giménez's result settings.

\section{Preliminaries}

Our main tool to build the proof of Theorem 4 is the following (and important) version of the Omori-Yau Maximum Principle for the Hessian due to Pigola, Rigoli and Setti [21, Theorem 1.9]. Chronologically, the Omori-Yau Maximum Principle can be traced in a series of papers, starting with Omori [18] who proved the maximum principle at infinity for the class of complete Riemannian manifolds with sectional curvature bounded from below, followed by new insights given by Cheng and Yau [5], 25], who extended them to the class of complete Riemannian manifolds with Ricci curvature bounded from below, and by extensions due to C. Dias [8] (extended to the class of sectional curvature with quadratic decay) and ChenXin 4 (extended to the class of Ricci curvature with quadratic decay). Finally, as observed by Pigola, Rigoli and Setti in [21, the validity of the Omori-Yau Maximum Principle does not depend on curvature bounds as much as one would expect. Actually, a condition to guarantee the validity of it can be expressed in a function theoretic form by the newest elaboration stated below.

Theorem 9 ([21, Theorem 1.9]). Let $M^{m}$ be a Riemannian manifold and assume that there exists a non-negative $C^{2}$-function $\psi$ satisfying the following requirements:

(a.1) $\psi$ is proper, that is, $\psi(x) \rightarrow+\infty$ as $x \rightarrow \infty$;

(a.2) there exists a positive constant $A>0$ such that $|\operatorname{grad} \psi| \leq A \sqrt{\psi}$ outside a compact subset of $M$;

(a.3) there exists a positive constant $B>0$ such that

$$
\text { Hess } \psi \leq B \sqrt{\psi G(\sqrt{\psi})}\langle,\rangle
$$

(in the sense of quadratic forms) outside a compact subset of $M$, where $G$ is a smooth function on $[0,+\infty)$ satisfying:

$$
\begin{array}{ll}
\text { (i) } G(0)>0, & \text { (ii) } G^{\prime}(t) \geq 0 \text { on }[0,+\infty), \\
\text { (iii) } 1 / \sqrt{G(t)} \notin L^{1}(0,+\infty), & \text { (iv) } \lim \sup _{t \rightarrow+\infty} \frac{t G(\sqrt{t})}{G(t)}<+\infty .
\end{array}
$$

Then, given a function $u \in C^{2}(M)$ with $u^{*}=\sup _{M} u<+\infty$ there exists a sequence $\left\{x_{k}\right\}_{k \in \mathbb{N}} \subset M$ such that

$$
\text { (i) } u\left(x_{k}\right)>u^{*}-\frac{1}{k} \text {, (ii) }\left|\operatorname{grad} u\left(x_{k}\right)\right|<\frac{1}{k} \text {, and (iii) } \operatorname{Hess} u\left(x_{k}\right)<\frac{1}{k}\langle,\rangle
$$

in the sense of quadratic forms, that is,

$$
\operatorname{Hess} u\left(x_{k}\right)(v, v)<\frac{1}{k}|v|^{2} \text { for all } v \in T_{x_{k}} M \text {. }
$$


If, instead of (a.3), one replaces it by

(a.3) $)^{\prime}$ there exists a positive constant $B>0$ such that $\Delta \gamma \leq B \sqrt{\gamma G(\sqrt{\gamma})}$ outside a compact subset of $M$,

then one can weaken conclusion (iii) in (2.2) to

$$
(\text { iii })^{\prime} \Delta u\left(x_{k}\right)<\frac{1}{k}
$$

Remark 10. It is worth pointing out that although in the statement (and in the proof) of Theorem 9 the manifold $M$ is not required to be geodesically complete, the two assumptions (a.1) and (a.2) imply it. For the details, see [21, page 10].

For that reason, and following the terminology introduced by Pigola, Rigoli and Setti in 21, the Omori-Yau Maximum Principle for the Hessian is said to hold on a (not necessarily complete) Riemannian manifold $M$ if, for any smooth funtion $u \in C^{2}(M)$ with $u^{*}=\sup _{M} u<+\infty$, there exists a sequence of points $\left\{x_{k}\right\}_{k \in \mathbb{N}} \subset M$ satisfying

$$
\text { (i) } u\left(x_{k}\right)>u^{*}-\frac{1}{k} \text {, (ii) }\left|\operatorname{grad} u\left(x_{k}\right)\right|<\frac{1}{k} \text {, and (iii) } \operatorname{Hess} u\left(x_{k}\right)<\frac{1}{k}\langle,\rangle
$$

for each $k \in \mathbb{N}$. Equivalently, for any smooth funtion $u \in C^{2}(M)$ with $u_{*}=$ $\inf _{M} u>-\infty$ there exists a sequence of points $\left\{x_{k}\right\}_{k \in \mathbb{N}} \subset M$ with the properties

$$
\text { (i) } u\left(x_{k}\right)<u_{*}+\frac{1}{k} \text {, (ii) }\left|\operatorname{grad} u\left(x_{k}\right)\right|<\frac{1}{k} \text {, and (iii) } \operatorname{Hess} u\left(x_{k}\right)>-\frac{1}{k}\langle,\rangle
$$

for each $k \in \mathbb{N}$. In the same way, the Omori-Yau Maximum Principle is said to hold on a (not necessarily complete) Riemannian manifold $M$ if, for any smooth function $u \in C^{2}(M)$ with $u^{*}=\sup _{M} u<+\infty$, there exists a sequence of points $\left\{x_{k}\right\}_{k \in \mathbb{N}} \subset M$ satisfying

$$
\text { (i) } u\left(x_{k}\right)>u^{*}-\frac{1}{k} \text {, (ii) }\left|\operatorname{grad} u\left(x_{k}\right)\right|<\frac{1}{k} \text {, and (iii) } \Delta u\left(x_{k}\right)<\frac{1}{k}
$$

for each $k \in \mathbb{N}$.

The function theoretic approach to the Omori-Yau Maximum Principle given in Theorem 9 allows one to apply it in different situations, where the choice of $\psi$ and $G$ are suggested by the geometric setting. For instance, one has the following consequence (see [21, Example 1.13]).

Corollary 11. Let $M$ be a complete, non-compact, Riemannian manifold, let $o \in$ $M$ be a reference point and denote by $\varrho_{M}(x)$ the Riemannian distance function from $o$. Assume that the radial sectional curvature of $M$, that is, the sectional curvature of the 2-planes containing grad $\varrho_{M}$, satisfies

$$
K_{M}^{r a d} \geq-G(r),
$$

where $G$ is a smooth function on $[0,+\infty)$ which we assume to be even at the origin, that is, $G^{(2 k+1)}(0)=0$ for $k=0,1,2, \ldots$, and satisfying the conditions listed in Theorem 9. Then the Omori-Yau Maximum Principle for the Hessian holds on M.

We will need two more results. The first is known as Otsuki's Lemma (for a proof see, for instance, [15, Page 28] or [21, Lemma 1.16]). 
Lemma 12. Let $\beta: \mathbb{R}^{k} \times \mathbb{R}^{k} \rightarrow \mathbb{R}^{q}, q \leq k-1$, be a bilinear symmetric form satisfying $\beta(X, X) \neq 0$ for $X \neq 0$. Then there exist linearly independent vectors $X, Y$ such that

$$
\beta(X, X)=\beta(Y, Y) \quad \text { and } \quad \beta(X, Y)=0 .
$$

The second is the well-known Hessian Comparison Theorem; see [22].

Theorem 13 (Hessian Comparison Theorem). Let $M$ be a Riemannian manifold and $x_{0}, x_{1} \in M$ be such that there is a minimizing unit speed geodesic $\gamma$ joining $x_{0}$ and $x_{1}$, and let $\varrho(x)=\operatorname{dist}\left(x_{0}, x\right)$ be the distance function to $x_{0}$. Let $K_{\gamma} \leq b$ be the radial sectional curvatures of $M$ along $\gamma$. If $b>0$ assume $\varrho\left(x_{1}\right)<\pi / 2 \sqrt{b}$. Then, we have

$$
\text { Hess } \varrho(x)\left(\gamma^{\prime}, \gamma^{\prime}\right)=0
$$

and

$$
\text { Hess } \varrho(x)(X, X) \geq C_{b}(\varrho(x))|X|^{2},
$$

where $X \in T_{x} M$ is perpendicular to $\gamma^{\prime}(\varrho(x))$.

\section{Proof of Theorem 4}

Let $\varphi: M^{m} \rightarrow \bar{M}^{n}$ be an isometric immersion between Riemannian manifolds. Given a function $g \in C^{\infty}(\bar{M})$ we set $f=g \circ \varphi \in C^{\infty}(M)$. Since

$$
\left\langle\operatorname{grad}^{M} f, X\right\rangle=\left\langle\operatorname{grad}^{\bar{M}} g, X\right\rangle
$$

for every vector field $X \in T M$, we obtain

$$
\operatorname{grad}^{\bar{M}} g=\operatorname{grad}^{M} f+\left(\operatorname{grad}^{\bar{M}} g\right)^{\perp}
$$

according to the decomposition $T \bar{M}=T M \oplus T^{\perp} M$. An easy computation using the Gauss formula gives the well-known relation (see e.g. [13])

$$
\operatorname{Hess}^{M} f(X, Y)=\operatorname{Hess}^{\bar{M}} g(X, Y)+\left\langle\operatorname{grad}^{\bar{M}} g, \alpha(X, Y)\right\rangle
$$

for all vector fields $X, Y \in T M$, where $\alpha$ stands for the second fundamental form of $\varphi$. In particular, taking traces with respect to an orthonormal frame $\left\{e_{1}, \ldots, e_{m}\right\}$ in $T M$ yields

$$
\Delta_{M} f=\sum_{i=1}^{m} \operatorname{Hess}^{\bar{M}} g\left(e_{i}, e_{i}\right)+\left\langle\operatorname{grad}^{\bar{M}} g, \vec{H}\right\rangle,
$$

where $\vec{H}=\sum_{i=1}^{m} \alpha\left(e_{i}, e_{i}\right)$.

3.1. Proof of Theorem 4, item (i). Let $g: N \times \mathbb{R}^{\ell} \rightarrow \mathbb{R}$ be given as $g(z, y)=$ $\phi_{b}\left(\varrho_{N}(z)\right)$, where

$$
\phi_{b}(t)= \begin{cases}1-\cos (\sqrt{b} t) & \text { if } b>0 \text { and } 0<t<\pi / 2 \sqrt{b}, \\ t^{2} & \text { if } b=0 \text { and } t>0 \\ \cosh (\sqrt{-b} t) & \text { if } b<0 \text { and } t>0\end{cases}
$$

and $\varrho_{N}(z)=\operatorname{dist}_{N}(p, z)$. Consider $f: M \rightarrow \mathbb{R}$ to be the function $f=g \circ \varphi$, and let $\pi_{N}: N \times \mathbb{R}^{\ell} \rightarrow N$ be the projection on the factor $N$. Since $\pi_{N}(\varphi(M)) \subset B_{N}(r)$, we have that $f^{*}=\sup _{M} f \leq \phi_{b}(r)<+\infty$. The idea of the proof is similar to the idea of Jorge-Koutrofiotis in [13]. We will need to apply the Omori-Yau Maximum Principle for the Hessian to the function $f$ in order to control the second 
fundamental form of the immersion restricted to a certain subspace and apply Otsuki's Lemma in the estimate of the sectional curvature.

To see that the Omori-Yau Maximum Principle for the Hessian holds on $M$, we may suppose that $\sup K_{M}<\infty$. Otherwise the estimate in (1.5) is trivially satisfied. In that case, since the scalar curvature is an average of sectional curvatures, it follows from (1.2) that

$$
K_{M}^{\mathrm{rad}}(x) \geq-\widehat{B}^{2} \varrho_{M}^{2}(x) \cdot \prod_{j=1}^{k}\left(\log ^{(j)}\left(\varrho_{M}(x)\right)\right)^{2}, \varrho_{M}(x) \gg 1,
$$

for a positive constant $\widehat{B}>0$, where $K_{M}^{\mathrm{rad}}$ denotes the radial sectional curvature of $M$. According to Corollary 11, this curvature decay suffices to conclude that the Omori-Yau Maximum Principle for the Hessian holds on $M$. Therefore, there exists a sequence of points $\left\{x_{k}\right\}$ in $M$ such that

$$
\text { (i) } f\left(x_{k}\right)>f^{*}-\frac{1}{k} \text {, (ii) }\left|\operatorname{grad} f\left(x_{k}\right)\right|<\frac{1}{k} \text {, and (iii) } \operatorname{Hess} f\left(x_{k}\right)<\frac{1}{k}\langle,\rangle \text {. }
$$

Since $f(x)=g(\varphi(x))=\phi_{b}\left(\varrho_{N}(z)\right)$, where $z=z(x)=\pi_{N}(\varphi(x))$, then

$$
\operatorname{grad}^{N \times \mathbb{R}^{\ell}} g(\varphi(x))=\operatorname{grad} f(x)+\left(\operatorname{grad}^{N \times \mathbb{R}^{\ell}} g(\varphi(x))\right)^{\perp},
$$

where

$$
\operatorname{grad}^{N \times \mathbb{R}^{\ell}} g(z, y)=\phi_{b}^{\prime}\left(\varrho_{N}(z)\right) \operatorname{grad}^{N} \varrho_{N}(z) .
$$

It then follows from (3.1) that

$$
\begin{aligned}
\text { Hess } f(x)(X, X)= & \operatorname{Hess}^{N}\left(\phi_{b} \circ \varrho_{N}\right)(z)\left(\pi_{T N} X, \pi_{T N} X\right) \\
& +\left\langle\operatorname{grad}^{N}\left(\phi_{b} \circ \varrho_{N}\right)(z), \alpha_{x}(X, X)\right\rangle
\end{aligned}
$$

for all vector fields $X \in T_{x} M$, where $\pi_{T N}$ denotes the orthogonal projection of $T M$ onto $T N$. Observe also that

$$
\begin{aligned}
\operatorname{Hess}^{N}\left(\phi_{b} \circ \varrho_{N}\right)(z)\left(\pi_{T N} X, \pi_{T N} X\right)= & \phi_{b}^{\prime \prime}(s)\left(\frac{\partial \varrho_{N}}{\partial X}\right)^{2} \\
& +\phi_{b}^{\prime}(s) \operatorname{Hess}^{N} \varrho_{N}(z)\left(\pi_{T N} X, \pi_{T N} X\right),
\end{aligned}
$$

where $s=s(x)=\varrho_{N}(z)$ and

$$
\frac{\partial \varrho_{N}}{\partial X}=\left\langle\operatorname{grad}^{N} \varrho_{N}, X\right\rangle .
$$

Taking into account that the function $\phi_{b}(t)$ satisfies the differential equation

$$
\phi_{b}^{\prime \prime}(t)-\phi_{b}^{\prime}(t) C_{b}(t)=0,
$$

it follows from (3.5) and (3.6) that

$$
\begin{aligned}
\text { Hess } f(x)(X, X)= & \phi_{b}^{\prime}(s)\left(C_{b}(s)\left(\frac{\partial \varrho_{N}}{\partial X}\right)^{2}+\left\langle\operatorname{grad}^{N} \varrho_{N}(z), \alpha_{x}(X, X)\right\rangle\right) \\
& +\phi_{b}^{\prime}(s) \operatorname{Hess}^{N} \varrho_{N}(z)\left(\pi_{T N} X, \pi_{T N} X\right) .
\end{aligned}
$$

Since $m \geq \ell+2$, we have for each $x \in M$ a subspace $V_{x} \subset T_{x} M \subset T_{\varphi(x)}\left(N \times \mathbb{R}^{\ell}\right)$ such that $V_{x} \perp T \mathbb{R}^{\ell}$ and $\operatorname{dim} V_{x} \geq m-\ell \geq 2$. Choose $\left\{\partial / \partial \varrho_{N}, \partial / \partial \theta_{2}, \ldots, \partial / \partial \theta_{n-\ell}\right\}$ 
orthonormal polar coordinates for $T N$. Then, for every $X \in V_{x}$ we have $\pi_{T \mathbb{R}^{\ell}} X=0$ and

$$
X=a_{1}^{X} \frac{\partial}{\partial \varrho_{N}}+\sum_{j=2}^{n-\ell} a_{j}^{X} \frac{\partial}{\partial \theta_{j}},
$$

where $a_{1}^{X}=\partial \varrho_{N} / \partial X$. Therefore, using Theorem 13, we have that for every $X \in V_{x}$

$$
\begin{aligned}
\operatorname{Hess}^{N} \varrho_{N}(z)\left(\pi_{T N} X, \pi_{T N} X\right) & =\sum_{j=2}^{n-\ell}\left(a_{j}^{X}\right)^{2} \operatorname{Hess}^{N} \varrho_{N}(z)\left(\frac{\partial}{\partial \theta_{j}}, \frac{\partial}{\partial \theta_{j}}\right) \\
& \geq C_{b}(s)\left(\langle,\rangle-d \varrho_{N} \otimes d \varrho_{N}\right)(X, X) \\
& =C_{b}(s)\left(|X|^{2}-\left(a_{1}^{X}\right)^{2}\right),
\end{aligned}
$$

since $\pi_{T N} X=X$, so that by (3.7) we have

$$
\begin{aligned}
\operatorname{Hess} f(x)(X, X) & \geq \phi_{b}^{\prime}(s)\left(C_{b}(s)|X|^{2}+\left\langle\operatorname{grad}^{N} \varrho_{N}(z), \alpha_{x}(X, X)\right\rangle\right) \\
& \geq \phi_{b}^{\prime}(s)\left(C_{b}(s)|X|^{2}-\left|\alpha_{x}(X, X)\right|\right)
\end{aligned}
$$

for every $X \in V_{x}$. From here and (3.3) we obtain that

$$
\frac{1}{k}|X|^{2} \geq \operatorname{Hess} f\left(x_{k}\right)(X, X) \geq \phi_{b}^{\prime}\left(s_{k}\right)\left(C_{b}\left(s_{k}\right)|X|^{2}-\left|\alpha_{x_{k}}(X, X)\right|\right)
$$

for every $x_{k}$ and every $X \in V_{x_{k}}$, where $z_{k}=\pi_{N}\left(\varphi\left(x_{k}\right)\right.$ and $s_{k}=s\left(x_{k}\right)=\varrho_{N}\left(z_{k}\right)$. Hence

$$
\left|\alpha_{x_{k}}(X, X)\right| \geq\left(C_{b}\left(s_{k}\right)-\frac{1}{k \phi_{b}^{\prime}\left(s_{k}\right)}\right)|X|^{2}
$$

with

$$
C_{b}\left(s_{k}\right)-\frac{1}{k \phi_{b}^{\prime}\left(s_{k}\right)}>0
$$

for $k$ sufficiently large.

Now consider $\beta_{x_{k}}: V_{x_{k}} \times V_{x_{k}} \rightarrow \mathbb{R}^{n-m}$ as the restriction of the second fundamental form $\alpha_{x_{k}}$ to $V_{x_{k}}$. We have that

$$
n-m \leq m-\ell-1 \leq \operatorname{dim} V_{x_{k}}-1
$$

since $2 m-1 \geq n+\ell$, and therefore we may apply Lemma 12 to $\beta_{x_{k}}$. The conclusion is that there are linearly independent vectors $X_{k}, Y_{k} \in V_{x_{k}}$ such that

$$
\alpha\left(X_{k}, X_{k}\right)=\alpha\left(Y_{k}, Y_{k}\right) \text { and } \alpha\left(X_{k}, Y_{k}\right)=0
$$

and $\left|X_{k}\right| \geq\left|Y_{k}\right| \geq 1$. We will now compare the sectional curvature $K_{M}\left(X_{k}, Y_{k}\right)$ in $M$ of the plane spanned by $X_{k}$ and $Y_{k}$ with the sectional curvature $K_{N \times \mathbb{R}^{\ell}}\left(X_{k}, Y_{k}\right)$ in $N \times \mathbb{R}^{\ell}$ of the same plane. Since $X_{k}, Y_{k} \in V_{x_{k}} \perp T \mathbb{R}^{\ell}$, then

$$
K_{N \times \mathbb{R}^{\ell}}\left(X_{k}, Y_{k}\right)=K_{N}\left(X_{k}, Y_{k}\right) .
$$


Then, by the Gauss equation, we have that

$$
\begin{aligned}
K_{M}\left(X_{k}, Y_{k}\right)-K_{N}\left(X_{k}, Y_{k}\right) & =K_{M}\left(X_{k}, Y_{k}\right)-K_{N \times \mathbb{R}^{\ell}}\left(X_{k}, Y_{k}\right) \\
& =\frac{\left\langle\alpha\left(X_{k}, X_{k}\right), \alpha\left(Y_{k}, Y_{k}\right)\right\rangle-\left|\alpha\left(X_{k}, Y_{k}\right)\right|^{2}}{\left|X_{k}\right|^{2}\left|Y_{k}\right|^{2}-\left\langle X_{k}, Y_{k}\right\rangle^{2}} \\
& \geq \frac{\left|\alpha\left(X_{k}, X_{k}\right)\right|^{2}}{\left|X_{k}\right|^{2}\left|Y_{k}\right|^{2}} \geq\left(\frac{\left|\alpha\left(X_{k}, X_{k}\right)\right|}{\left|X_{k}\right|^{2}}\right)^{2} \\
& \geq\left(C_{b}\left(s_{k}\right)-\frac{1}{k \phi_{b}^{\prime}\left(s_{k}\right)}\right)^{2} \cdot
\end{aligned}
$$

Thus

$$
\sup _{M} K_{M}-\inf _{B_{N}(r)} K_{N} \geq\left(C_{b}\left(s_{k}\right)-\frac{1}{k \phi_{b}^{\prime}\left(s_{k}\right)}\right)^{2}
$$

Observe that $f^{*}=\phi_{b}\left(s^{*}\right)$, where $s^{*}=\sup _{M} s$ and $s_{k} \rightarrow s^{*} \leq r$. Therefore, letting $k \rightarrow \infty$ we have that

$$
\sup _{M} K_{M}-\inf _{B_{N}(r)} K_{N} \geq C_{b}^{2}\left(s^{*}\right) \geq C_{b}^{2}(r) .
$$

This finishes the proof of item (i) in Theorem 4

3.2. Proof of Theorem 4, item (ii). In this case, we cannot directly apply Theorem 9 but we may apply parts of its proof. Again consider $f: M \rightarrow \mathbb{R}$ as the function given by $f(x)=\phi_{b}\left(\varrho_{N}(z(x))\right)$, with $z(x)=\pi_{N}(\varphi(x))$. Since $\pi_{N}(\varphi(M)) \subset$ $B_{N}(r)$, we have that $f^{*}=\sup _{M} f \leq \phi_{b}(r)$. Let $\psi: M \rightarrow[0,+\infty)$ be given by

$$
\psi(x)=\exp \left(\int_{0}^{|y(x)|} \frac{d s}{\sigma(s)}\right),
$$

where $y(x)=\pi_{\mathbb{R}^{\ell}}(\varphi(x))$. Since $\varphi$ is proper and $\pi_{N}(\varphi(M)) \subset B_{N}(r)$, then the function $|y(x)|$ satisfies $|y(x)| \rightarrow+\infty$ as $x \rightarrow \infty$. By hypothesis we have that $\int_{0}^{+\infty} 1 / \sigma(s) d s=+\infty$ so that $\psi(x) \rightarrow+\infty$ as $x \rightarrow \infty$.

Following the ideas of Pigola, Rigoli and Setti in the proof of [21, Theorem 1.9], we let $x_{0} \in M$ with $\pi_{N}\left(\varphi\left(x_{0}\right)\right) \neq p$ and set

$$
f_{k}(x)=\frac{f(x)-f\left(x_{0}\right)+1}{\psi(x)^{1 / k}} .
$$

Thus $f_{k}\left(x_{0}\right)>0$, and since $f^{*} \leq \phi_{b}(r)<+\infty$ and $\psi(x) \rightarrow+\infty$ as $x \rightarrow \infty$, we have that $\limsup _{x \rightarrow \infty} f_{k}(x) \leq 0$. Hence $f_{k}$ attains a positive absolute maximum at a point $x_{k} \in M$. This procedure yields a sequence $\left\{x_{k}\right\}$ such that (passing to a subsequence if necessary) $f\left(x_{k}\right)$ converges to $f^{*}$ (see page 8 of [21]).

First suppose that $x_{k} \rightarrow \infty$ as $k \rightarrow+\infty$. Since $f_{k}$ attains a positive maximum at $x_{k}$, we have grad $f_{k}\left(x_{k}\right)=0$ and Hess $f_{k}\left(x_{k}\right)(X, X) \leq 0$ for every $X \in T_{x_{k}} M$. This yields

$$
\operatorname{grad} f\left(x_{k}\right)=\frac{f\left(x_{k}\right)-f\left(x_{0}\right)+1}{k \psi\left(x_{k}\right)} \operatorname{grad} \psi\left(x_{k}\right)
$$


and

$$
\begin{aligned}
\operatorname{Hess} f\left(x_{k}\right) & \leq \frac{f\left(x_{k}\right)-f\left(x_{0}\right)+1}{k \psi\left(x_{k}\right)}\left(\operatorname{Hess} \psi\left(x_{k}\right)+\left(\frac{1}{k}-1\right) \frac{1}{\psi\left(x_{k}\right)} d \psi \otimes d \psi\right) \\
& \leq \frac{f\left(x_{k}\right)-f\left(x_{0}\right)+1}{k \psi\left(x_{k}\right)} \operatorname{Hess} \psi\left(x_{k}\right) .
\end{aligned}
$$

Since $\psi(x)=\zeta(y)$, where $y=y(x)$ and $\zeta(y)=\exp \left(\int_{0}^{|y|} d s / \sigma(s)\right), y \in \mathbb{R}^{\ell}$, from (3.1) we have that

$$
\operatorname{Hess} \psi(x)(X, X)=\operatorname{Hess}^{\mathbb{R}^{\ell}} \zeta(y)\left(\pi_{T \mathbb{R}^{\ell}} X, \pi_{T \mathbb{R}^{\ell}} X\right)+\left\langle\operatorname{grad}^{\mathbb{R}^{\ell}} \zeta(y), \alpha_{x}(X, X)\right\rangle
$$

for all vectors $X \in T_{x} M$, where $\pi_{T \mathbb{R}^{\ell}}$ denotes the orthogonal projection of $T M$ onto $T \mathbb{R}^{\ell}$. Also observe that

$$
\operatorname{grad}^{\mathbb{R}^{\ell}} \zeta(y)=\frac{\zeta(y)}{\sigma(|y|)} \operatorname{grad}^{\mathbb{R}^{\ell}}|y|
$$

and then

$$
\operatorname{grad} \psi(x)=\frac{\psi(x)}{\sigma(|y|)} \operatorname{grad}^{\mathbb{R}^{\ell}}|y| .
$$

Thus, for every $X \in T_{x} M$ such that $\pi_{T \mathbb{R}^{\ell}} X=0$, it follows from (3.12) that

$$
\operatorname{Hess} \psi(x)(X, X)=\frac{\psi(x)}{\sigma(|y(x)|)}\left\langle\operatorname{grad}^{\mathbb{R}^{\ell}}|y|, \alpha_{x}(X, X)\right\rangle \leq \frac{\psi(x)}{\sigma(|y(x)|)}\left|\alpha_{x}(X, X)\right| .
$$

Therefore, by (1.4) we obtain that

$$
\frac{1}{\psi(x)} \operatorname{Hess} \psi(x)(X, X) \leq \frac{\left|\alpha_{x}(X, X)\right|}{\sigma(|y(x)|)} \leq|X|^{2}
$$

for every $X \in T_{x} M$ with $\pi_{T \mathbb{R}^{\ell}} X=0$.

As in the proof of item (i), since $m \geq \ell+2$, we may choose for each $x_{k} \in M$ a subspace $V_{x_{k}} \subset T_{x_{k}} M$ with $\operatorname{dim} V_{x_{k}} \geq m-\ell \geq 2$ and such that $V_{x_{k}} \perp T \mathbb{R}^{\ell}$. Then, $\pi_{T \mathbb{R}^{\ell}} X=0$ for every $X \in V_{x_{k}}$, and from (3.11) and (3.14) we get that

$$
\text { Hess } f\left(x_{k}\right)(X, X) \leq \frac{f\left(x_{k}\right)-f\left(x_{0}\right)+1}{k \psi\left(x_{k}\right)} \operatorname{Hess} \psi\left(x_{k}\right)(X, X) \leq \frac{\phi_{b}(r)+1}{k}|X|^{2},
$$

for every $X \in V_{x}$. Moreover, using Theorem 13 we also have here that

$$
\operatorname{Hess} f(x)(X, X) \geq \phi_{b}^{\prime}(s)\left(C_{b}(s)|X|^{2}-\left|\alpha_{x}(X, X)\right|\right)
$$

for every $X \in V_{x}$, since $\pi_{T N} X=X$. Therefore, we obtain that

$$
\frac{\phi_{b}(r)+1}{k}|X|^{2} \geq \operatorname{Hess} f\left(x_{k}\right)(X, X) \geq \phi_{b}^{\prime}\left(s_{k}\right)\left(C_{b}\left(s_{k}\right)|X|^{2}-\left|\alpha_{x_{k}}(X, X)\right|\right)
$$

for every $x_{k}$ and every $X \in V_{x_{k}}$, where $z_{k}=\pi_{N}\left(\varphi\left(x_{k}\right)\right)$ and $s_{k}=s\left(x_{k}\right)=\varrho_{N}\left(z_{k}\right)$. Hence

$$
\left|\alpha_{x_{k}}(X, X)\right| \geq\left(C_{b}\left(s_{k}\right)-\frac{\phi_{b}(r)+1}{k \phi_{b}^{\prime}\left(s_{k}\right)}\right)|X|^{2}
$$

with

$$
C_{b}\left(s_{k}\right)-\frac{\phi_{b}(r)+1}{k \phi_{b}^{\prime}\left(s_{k}\right)}>0
$$


for $k$ sufficiently large. Reasoning now as in the last part of the proof of item (i), there exist linearly independent vectors $X_{k}, Y_{k} \in V_{x_{k}}$ such that, by Gauss' equation,

$$
K_{M}\left(X_{k}, Y_{k}\right)-K_{N}\left(X_{k}, Y_{k}\right)=\left(\frac{\left|\alpha\left(X_{k}, X_{k}\right)\right|}{\left|X_{k}\right|^{2}}\right)^{2} \geq\left(C_{b}\left(s_{k}\right)-\frac{\phi_{b}(r)+1}{k \phi_{b}^{\prime}\left(s_{k}\right)}\right)^{2} .
$$

We obtain from here that

$$
\sup _{M} K_{M}-\inf _{B_{N}(r)} K_{N} \geq\left(C_{b}\left(s_{k}\right)-\frac{\phi_{b}(r)+1}{k \phi_{b}^{\prime}\left(s_{k}\right)}\right)^{2},
$$

and letting $k \rightarrow \infty$ we conclude that

$$
\sup _{M} K_{M}-\inf _{B_{N}(r)} K_{N} \geq C_{b}^{2}\left(s^{*}\right) \geq C_{b}^{2}(r),
$$

where $s^{*}=\sup _{M} s, f^{*}=\phi_{b}\left(s^{*}\right)$ and $s_{k} \rightarrow s^{*} \leq r$.

To finish the proof of item (ii), we need to consider the case where the sequence $\left\{x_{k}\right\} \subset M$ remains in a compact set. In that case, passing to a subsequence if necessary, we may assume that $x_{k} \rightarrow x_{\infty} \in M$ and $f$ attains its absolute maximum at $x_{\infty}$. Thus Hess $f\left(x_{\infty}\right)(X, X) \leq 0$ for all $X \in T_{x_{\infty}} M$. In particular, it follows from (3.16) that for every $X \in V_{x_{\infty}}$

$$
0 \geq \operatorname{Hess} f\left(x_{\infty}\right)(X, X) \geq \phi_{b}^{\prime}\left(s_{\infty}\right)\left(C_{b}\left(s_{\infty}\right)|X|^{2}-\left|\alpha_{x_{\infty}}(X, X)\right|\right),
$$

where $s_{\infty}=\varrho_{N}\left(\pi_{N}\left(\varphi\left(x_{\infty}\right)\right)\right)$. Therefore

$$
\left|\alpha_{x_{\infty}}(X, X)\right| \geq C_{b}\left(s_{\infty}\right)|X|^{2} .
$$

By applying Lemma 12 to $\beta_{x_{\infty}}: V_{x_{\infty}} \times V_{x_{\infty}} \rightarrow \mathbb{R}^{n-m}$, the restriction of the second fundamental form $\alpha_{x_{\infty}}$ to $V_{x_{\infty}}$, and reasoning again as in the last part of the proof of item (i), we have that there exist linearly independent vectors $X_{\infty}, Y_{\infty} \in V_{x_{\infty}}$ such that, by Gauss' equation,

$$
K_{M}\left(X_{\infty}, Y_{\infty}\right)-K_{N}\left(X_{\infty}, Y_{\infty}\right)=\left(\frac{\left|\alpha\left(X_{\infty}, X_{\infty}\right)\right|}{\left|X_{\infty}\right|^{2}}\right)^{2} \geq C_{b}^{2}\left(s_{\infty}\right) .
$$

Thus, we conclude from here that

$$
\sup _{M} K_{M}-\inf _{B_{N}(r)} K_{N} \geq C_{b}^{2}\left(s_{\infty}\right) \geq C_{b}^{2}(r) .
$$

This finishes the proof of Theorem 4

\section{Proof of Theorem 7}

We proceed as in the proof of Theorem 4 item (ii), to obtain a sequence $\left\{x_{k}\right\}$ such that $f\left(x_{k}\right)$ converges to $f^{*}$ and satisfying

$$
\operatorname{grad} f\left(x_{k}\right)=\frac{f\left(x_{k}\right)-f\left(x_{0}\right)+1}{k \psi\left(x_{k}\right)} \operatorname{grad} \psi\left(x_{k}\right)
$$

and

$$
\text { Hess } f\left(x_{k}\right) \leq \frac{f\left(x_{k}\right)-f\left(x_{0}\right)+1}{k \psi\left(x_{k}\right)} \operatorname{Hess} \psi\left(x_{k}\right) .
$$

Recall that (see (3.13)

$$
\operatorname{grad} \psi(x)=\frac{\psi(x)}{\sigma(|y|)} \operatorname{grad}^{\mathbb{R}^{\ell}}|y| .
$$


Let us first consider the case where $x_{k} \rightarrow \infty$ as $k \rightarrow+\infty$. From (4.1) and (4.3) we know that

$$
\left|\operatorname{grad} f\left(x_{k}\right)\right| \leq \frac{\left(f^{*}+1\right)}{k} \frac{1}{\sigma\left(\left|y_{k}\right|\right)} \leq \frac{\left(\phi_{b}(r)+1\right)}{k} \frac{1}{\sigma\left(\left|y_{k}\right|\right)},
$$

where $y_{k}=y\left(x_{k}\right)$. Since $\varphi$ is proper and $\pi_{N}(\varphi(M)) \subset B_{N}(r)$, then $\left|y_{k}\right| \rightarrow+\infty$ as $k \rightarrow+\infty$. Therefore, taking into account that $\lim \sup _{t \rightarrow+\infty} 1 / \sigma(t)<+\infty$ we obtain from here that

$$
\lim _{k \rightarrow+\infty}\left|\operatorname{grad} f\left(x_{k}\right)\right|=0 .
$$

Observe that

$$
\operatorname{grad}^{N \times \mathbb{R}^{\ell}} g(\varphi(x))=\phi_{b}^{\prime}\left(\varrho_{N}(z)\right) \operatorname{grad}^{N} \varrho_{N}(z)=\operatorname{grad} f(x)+\left(\operatorname{grad}^{N \times \mathbb{R}^{\ell}} g(\varphi(x))\right)^{\perp},
$$

where $z=z(x)=\pi_{N}(\varphi(x))$. Therefore,

$$
\phi_{b}^{\prime}\left(s_{k}\right)^{2}=\left|\operatorname{grad} f\left(x_{k}\right)\right|^{2}+\left|\left(\operatorname{grad}^{N \times \mathbb{R}^{\ell}} g\left(\varphi\left(x_{k}\right)\right)\right)^{\perp}\right|^{2},
$$

with $s_{k}=\varrho_{N}\left(z\left(x_{k}\right)\right)$, and making $k \rightarrow \infty$ here we obtain that

$$
\lim _{k \rightarrow+\infty}\left|\left(\operatorname{grad}^{N \times \mathbb{R}^{\ell}} g\left(\varphi\left(x_{k}\right)\right)\right)^{\perp}\right|=\phi_{b}^{\prime}\left(s^{*}\right)>0,
$$

which implies that

$$
\left(\operatorname{grad}^{N \times \mathbb{R}^{\ell}} g\left(\varphi\left(x_{k}\right)\right)\right)^{\perp} \neq 0
$$

for $k$ sufficiently large.

As in the proof of Theorem 4, since $m=n-1 \geq \ell+2$, we may choose for each $x_{k} \in M$ a subspace $V_{x_{k}} \subset T_{x_{k}} M$ with $\operatorname{dim} V_{x_{k}} \geq n-1-\ell \geq 2$ and such that $V_{x_{k}} \perp T \mathbb{R}^{\ell}$. Then, using Theorem[13, we also have that

$$
\text { Hess } f\left(x_{k}\right)(X, X) \geq \phi_{b}^{\prime}\left(s_{k}\right)\left(C_{b}\left(s_{k}\right)|X|^{2}-\left|\alpha_{x_{k}}(X, X)\right|\right)
$$

for every $X \in V_{x_{k}} \leq T_{x_{k}} M$, since $\pi_{T N} X=X$. On the other hand, we also know from (4.2) that

$$
\begin{aligned}
\text { Hess } f\left(x_{k}\right)(X, X) & \leq \frac{\left(\phi_{b}(r)+1\right)}{k} \frac{\operatorname{Hess} \psi\left(x_{k}\right)(X, X)}{\psi\left(x_{k}\right)} \\
& =\frac{\left(\phi_{b}(r)+1\right)}{k} \frac{1}{\sigma\left(\left|y_{k}\right|\right)}\left\langle\operatorname{grad}^{\mathbb{R}^{\ell}}|y|, \alpha_{x_{k}}(X, X)\right\rangle
\end{aligned}
$$

for every $X \in T_{x_{k}} M$. Since $m=n-1$ and $\left(\operatorname{grad}^{N \times \mathbb{R}^{\ell}} g\left(\varphi\left(x_{k}\right)\right)\right)^{\perp} \neq 0$ (for $k$ large enough), then

$$
\alpha_{x_{k}}(X, X)=\lambda_{x_{k}}(X, X)\left(\operatorname{grad}^{N \times \mathbb{R}^{\ell}} g\left(\varphi\left(x_{k}\right)\right)\right)^{\perp}
$$

for a real function $\lambda$.

Now observe that

$$
\begin{aligned}
\left\langle\operatorname{grad}^{\mathbb{R}^{\ell}}|y|, \alpha_{x_{k}}(X, X)\right\rangle & =\lambda_{x_{k}}(X, X)\left\langle\operatorname{grad}^{\mathbb{R}^{\ell}}|y|,\left(\operatorname{grad}^{N \times \mathbb{R}^{\ell}} g\left(\varphi\left(x_{k}\right)\right)\right)^{\perp}\right\rangle \\
& =\lambda_{x_{k}}(X, X)\left\langle\operatorname{grad}^{\mathbb{R}^{\ell}}|y|, \operatorname{grad} f\left(x_{k}\right)\right\rangle
\end{aligned}
$$

because of $\left\langle\operatorname{grad}^{\mathbb{R}^{\ell}}|y|, \operatorname{grad}^{N} \varrho_{N}\right\rangle=0$. Therefore,

$$
\begin{aligned}
\left\langle\operatorname{grad}^{\mathbb{R}^{\ell}}|y|, \alpha_{x_{k}}(X, X)\right\rangle & \leq\left|\lambda_{x_{k}}(X, X)\right|\left|\operatorname{grad} f\left(x_{k}\right)\right| \\
& \leq\left|\lambda_{x_{k}}(X, X)\right| \frac{\left(\phi_{b}(r)+1\right)}{k} \frac{1}{\sigma\left(\left|y_{k}\right|\right)} .
\end{aligned}
$$


On the other hand, from our hypothesis (1.9) we know that

$$
\left|\alpha_{x}(X, X)\right| \leq \sigma^{2}(|y(x)|)|X|^{2},
$$

and from (4.5) and (4.8) we have that

$$
\left|\alpha_{x_{k}}(X, X)\right|=\left|\lambda_{x_{k}}(X, X)\right| \sqrt{\phi_{b}^{\prime}\left(s_{k}\right)^{2}-\left|\operatorname{grad} f\left(x_{k}\right)\right|^{2}} \leq \sigma^{2}\left(\left|y_{k}\right|\right)|X|^{2} .
$$

That is,

$$
\frac{\left|\lambda_{x_{k}}(X, X)\right|}{\sigma\left(\left|y_{k}\right|\right)} \leq \frac{\sigma\left(\left|y_{k}\right|\right)|X|^{2}}{\sqrt{\phi_{b}^{\prime}\left(s_{k}\right)^{2}-\left|\operatorname{grad} f\left(x_{k}\right)\right|^{2}}} .
$$

It follows from here that

$$
\left\langle\operatorname{grad}^{\mathbb{R}^{\ell}}|y|, \alpha_{x_{k}}(X, X)\right\rangle \leq \frac{\left(\phi_{b}(r)+1\right)}{k} \frac{\sigma\left(\left|y_{k}\right|\right)|X|^{2}}{\sqrt{\phi_{b}^{\prime}\left(s_{k}\right)^{2}-\left|\operatorname{grad} f\left(x_{k}\right)\right|^{2}}}
$$

for every $X \in T_{x_{k}} M$, so that by (4.7) we get

$$
\text { Hess } f\left(x_{k}\right)(X, X) \leq \frac{\left(\phi_{b}(r)+1\right)^{2}}{k^{2}} \frac{|X|^{2}}{\sqrt{\phi_{b}^{\prime}\left(s_{k}\right)^{2}-\left|\operatorname{grad} f\left(x_{k}\right)\right|^{2}}} .
$$

Therefore, from (4.6) and (4.9) we have that

$$
\phi_{b}^{\prime}\left(s_{k}\right)\left(C_{b}\left(s_{k}\right)|X|^{2}-\left|\alpha_{x_{k}}(X, X)\right|\right) \leq \frac{\left(\phi_{b}(r)+1\right)^{2}}{k^{2}} \frac{|X|^{2}}{\sqrt{\phi_{b}^{\prime}\left(s_{k}\right)^{2}-\left|\operatorname{grad} f\left(x_{k}\right)\right|^{2}}}
$$

for every $X \in V_{x_{k}}$. Hence

$$
\left|\alpha_{x_{k}}(X, X)\right| \geq\left(C_{b}\left(s_{k}\right)-\frac{\left(\phi_{b}(r)+1\right)^{2}}{k^{2} \phi_{b}^{\prime}\left(s_{k}\right) \sqrt{\phi_{b}^{\prime}\left(s_{k}\right)^{2}-\left|\operatorname{grad} f\left(x_{k}\right)\right|^{2}}}\right)|X|^{2},
$$

with

$$
\lim _{k \rightarrow+\infty}\left(C_{b}\left(s_{k}\right)-\frac{\left(\phi_{b}(r)+1\right)^{2}}{k^{2} \phi_{b}^{\prime}\left(s_{k}\right) \sqrt{\phi_{b}^{\prime}\left(s_{k}\right)^{2}-\left|\operatorname{grad} f\left(x_{k}\right)\right|^{2}}}\right)=C_{b}\left(s^{*}\right) \geq C_{b}(r)>0,
$$

where $s^{*}=\sup _{M} s, f^{*}=\phi_{b}\left(s^{*}\right)$ and $s_{k} \rightarrow s^{*} \leq r$. Reasoning now as in the last part of the proof of item (i), there exist linearly independent vectors $X_{k}, Y_{k} \in V_{x_{k}}$ such that, by Gauss' equation,

$$
\begin{aligned}
K_{M}\left(X_{k}, Y_{k}\right)-K_{N}\left(X_{k}, Y_{k}\right) & =\left(\frac{\left|\alpha\left(X_{k}, X_{k}\right)\right|}{\left|X_{k}\right|^{2}}\right)^{2} \\
& \geq\left(C_{b}\left(s_{k}\right)-\frac{\left(\phi_{b}(r)+1\right)^{2}}{k^{2} \phi_{b}^{\prime}\left(s_{k}\right) \sqrt{\phi_{b}^{\prime}\left(s_{k}\right)^{2}-\left|\operatorname{grad} f\left(x_{k}\right)\right|^{2}}}\right) .
\end{aligned}
$$

We obtain from here that

$$
\sup _{M} K_{M}-\inf _{B_{N}(r)} K_{N} \geq\left(C_{b}\left(s_{k}\right)-\frac{\left(\phi_{b}(r)+1\right)^{2}}{k^{2} \phi_{b}^{\prime}\left(s_{k}\right) \sqrt{\phi_{b}^{\prime}\left(s_{k}\right)^{2}-\mid \operatorname{grad} f\left(x_{k}\right)^{2}}}\right)^{2},
$$

and letting $k \rightarrow \infty$ we conclude that

$$
\sup _{M} K_{M}-\inf _{B_{N}(r)} K_{N} \geq C_{b}^{2}\left(s^{*}\right) \geq C_{b}^{2}(r) .
$$

Finally, in the case where the sequence $\left\{x_{k}\right\} \subset M$ remains in a compact subset of $M$, and passing to a subsequence if necessary, we may assume that $x_{k} \rightarrow x_{\infty} \in M$ 
and $f$ attains its absolute maximum at $x_{\infty}$. Thus, Hess $f\left(x_{\infty}\right)(X, X) \leq 0$ for all $X \in T_{x_{\infty}} M$. Therefore, it follows again from Theorem 13 that for every $X \in V_{x_{\infty}}$,

$$
0 \geq \operatorname{Hess} f\left(x_{\infty}\right)(X, X) \geq \phi_{b}^{\prime}\left(s_{\infty}\right)\left(C_{b}\left(s_{\infty}\right)|X|^{2}-\left|\alpha_{x_{\infty}}(X, X)\right|\right),
$$

where $s_{\infty}=\varrho_{N}\left(\pi_{N}\left(\varphi\left(x_{\infty}\right)\right)\right)$ and $V_{x_{\infty}} \subset T_{x_{\infty}} M$ is a subspace with $\operatorname{dim} V_{x_{\infty}} \geq$ $n-1-\ell \geq 2$ and such that $V_{x_{\infty}} \perp T \mathbb{R}^{\ell}$. The proof now finishes as at the end of item (ii) in Theorem 4 .

\section{REFERENCES}

[1] L.J. Alías, G.P. Bessa and M. Dajczer, The mean curvature of cylindrically bounded submanifolds. Math. Ann. 345 (2009), 367-376. MR2529479 (2010i:53104)

[2] G. P. Bessa and J. F. Montenegro, On compact H-hypersurfaces of $N \times \mathbb{R}$. Geom. Dedicata 127 (2007), 1-5. MR2338510 (2008k:53110)

[3] E. Calabi, Problems in Differential Geometry (S. Kobayashi and J. Eells, Jr., eds.) Proc. of the United States-Japan Seminar in Differential Geometry, Kyoto, Japan, 1965, Nippon Hyoronsha Co. Ltd., Tokyo (1966) 170.

[4] Q. Chen and Y. L. Xin, A generalized maximum principle and its applications in geometry. Amer. J. of Math. 114 (1992), 355-366. MR1156569 (93g:53054)

[5] S. Y. Cheng and S. T. Yau, Differential equations on Riemannian manifolds and their applications. Comm. Pure. Appl. Math. 28 (1975), 333-354. MR0385749 (52:6608)

[6] S. S. Chern, The Geometry of G-structures. Bull. Amer. Math. Soc. 72 (1966), 167-219. MR0192436 (33:661)

[7] S. S. Chern and N. H. Kuiper, Some theorems on the isometric imbedding of compact Riemannian manifolds in Euclidean space. Ann. of Math. (2) 56 (1952), 442-430. MR0050962 $(14: 408 \mathrm{e})$

[8] C. C. Dias, Isometric immersions with slow growth of curvature. An. Acad. Bras. Ciênc. 54 (1982), 293-295. MR680268 (83k:53079)

[9] N. Efimov, Hyperbolic problems in the theory of surfaces. Proc. Int. Congress Math. Moscow (1966); Am. Math. Soc. Translation 70 (1968), 2638. MR0238232 (38:6508)

[10] F. Giménez, Estimates for the curvature of a submanifold which lies inside a tube. J. Geom. 58 (1997), 95-105. MR:1434182 (97k:53058)

[11] Th. Hasanis and D. Koutroufiotis, Immersions of Riemannian manifolds into cylinders. Arch. Math. 40 (1983), 82-85. MR720897 (85a:53047)

[12] D. Hilbert, Über Flächen von konstanter Krümmung. Trans. Amer. Math. Soc. 2 (1901), 87-99. MR1500557

[13] L. Jorge and D. Koutrofiotis, An estimate for the curvature of bounded submanifolds. Amer. J. Math. 103 (1980), 711-725. MR623135 (83d:53041b)

[14] L. Jorge, and F. Xavier, An inequality between the exterior diameter and the mean curvature of bounded immersions. Math. Z. 178 (1981), 77-82. MR.627095 (82k:53080)

[15] S. Kobayashi, K. Nomizu, Foundations of Differential Geometry, vol II. Interscience Tracts in Pure and Appl. Math., no. 15, New York (1969). MR0238225 (38:6501)

[16] J. D. Moore, An application of second variation to submanifold theory. Duke Math. J. 42 (1975), 191-193. MR0377776 (51:13945)

[17] J. Nash, The imbedding problem for Riemannian manifolds. Ann. of Math. (2) 63 (1956), 20-63. MR 0075639 (17:782b)

[18] H. Omori, Isometric immersions of Riemannian manifolds. J. Math. Soc. Japan 19 (1967), 205-214. MR0215259(35:6101)

[19] B. O'Neill, Immersions of manifolds of non-positive curvature. Proc. Amer. Math. Soc. 11 (1960), 132-134. MR117683(22:8459)

[20] T. Otsuki, Isometric imbedding of Riemannian manifolds in a Riemannian manifold. J. Math. Soc. Japan 6 (1954), 221-234. MR0067550 (16:747b)

[21] S. Pigola, M. Rigoli and A. Setti, Maximum Principle on Riemannian Manifolds ans Applications. Mem. Amer. Math. Soc. 174, no. 822 (2005). MR2116555 (2006b:53048)

[22] R. Schoen, and S. T. Yau, Lectures on Differential Geometry. Conference Proceedings and Lecture Notes in Geometry and Topology, vol. 1 (1994). MR1333601 (97d:53001) 
[23] E. Stiel, Immersions into manifolds of constant negative curvature. Proc. Amer. Math. Soc. 18 (1967), 713-715. MR0212743 (35:3609)

[24] C. Tompkins, Isometric embedding of flat manifolds in Euclidean spaces. Duke Math. J. 5 (1939), 58-61. MR 1546106

[25] S. T. Yau, Harmonic functions on complete Riemannian manifolds. Comm. Pure Appl. Math. 28 (1975), 201-228. MR0431040 (55:4042)

Departamento de Matemáticas, Universidad de Murcia, E-30100 Espinardo, Murcia, SPAIN

E-mail address: ljalias@um.es

Departamento de Matemática, Universidade Federal do Ceara-UFC, Bloco 914, Campus do Pici, 60455-760, Fortaleza, Ceara, Brazil

E-mail address: bessa@mat.ufc.br

Departamento de Matemática, Universidade Federal do Ceara-UFC, Bloco 914, Campus do Pici, 60455-760, Fortaleza, Ceara, Brazil

E-mail address: fabio@mat.ufc.br 